\title{
Targeting HSF1 leads to an antitumor effect in human epithelial ovarian cancer
}

\author{
YI-FEI CHEN ${ }^{1}$, SHU-YING WANG ${ }^{1}$, YOU-HUI YANG ${ }^{2}$, JIANG ZHENG $^{2}$, TING LIU $^{2,3}$ and LI WANG ${ }^{1}$ \\ ${ }^{1}$ The International Peace Maternity and Child Health Hospital, School of Medicine, Shanghai Jiaotong University, \\ Shanghai 200030; ${ }^{2}$ Guizhou Provincial Key Laboratory of Pharmaceutics, Guizhou Medical University; \\ ${ }^{3}$ National Engineering Research Center of Miao's Medicines, Guiyang, Guizhou 550004, P.R. China
}

Received August 22, 2016; Accepted April 21, 2017

DOI: $10.3892 /$ ijmm.2017.2978

\begin{abstract}
Late diagnosis and lack of specific therapeutic targets contribute to the low survival rate of patients with epithelial ovarian cancer (EOC), the most lethal gynecologic malignancy. Therefore, the screening of diagnostic markers and the identification of therapeutic targets are urgently required. Heat shock factor 1 (HSF1) has been demonstrated to be overexpressed in certain malignancies and to be involved in tumor initiation, development, transformation and metastasis. It is believed that HSF1 is a promising candidate for antitumor therapy. However, its expression pattern and function in ovarian cancer are far from being fully elucidated. Therefore, we examined the HSF1 expression in human EOC tissues, and evaluated its carcinogenesis-promoting activity in a xenograft tumor model. Examination of HSF1 expression in human EOC tissues was performed by immunohistochemical assay using ovarian tissue blots. Specific short hairpin RNA (shRNA) against HSF1 was employed to knockdown HSF1 in SKOV3 cells. Cell proliferative activity was evaluated by 3-(4,5-dimethylthiazol2-yl)-2,5-diphenyl-tetrazolium bromide (MTT) assay; cell cycle distribution and apoptosis were determined by flow cytometric analysis. In normal ovarian tissues, HSF1 was barely detected, whereas, high expression of HSF1 was found in malignant EOC tissues, including serous, mucinous, endometrioid, and clear cell EOC tissues. Suppressed proliferative activity and intensified apoptosis were observed in HSF1-knockdown SKOV3 cells. In nude mouse xenografts, downregulation of HSF1 was found to cause reduced carinogenesis, indicating the antitumor effect induced by modulation of HSF1 against EOC. Our findings suggest that HSF1 may be considered as a
\end{abstract}

Correspondence to: Dr Li Wang, The International Peace Maternity and Child Health Hospital, School of Medicine, Shanghai Jiaotong University, 910 Hengshan Road, Shanghai 200030, P.R. China E-mail: wangli_ivf@163.com

Dr Ting Liu, Guizhou Provincial Key Laboratory of Pharmaceutics, Guizhou Medical University, 9 Beijing Road, Guiyang, Guizhou 550004, P.R. China

E-mail: t-liu@163.com

Key words: human epithelial ovarian cancer, heat shock factor 1 potential candidate diagnostic marker of human EOC, and that modulation of HSF1 could be a promising therapeutic strategy against human EOC.

\section{Introduction}

Despite improvement in the scheduling and administration of chemotherapy against human epithelial ovarian cancer (EOC), EOC remains the most lethal gynecologic malignancy, and the 5 -year overall survival has achieved only a marginal improvement during the last decade $(1,2)$. EOC is the fifth leading cause of cancer-related death in women, at least partially due to late diagnosis and frequent chemotherapy resistance. The majority of EOC patients are diagnosed with locally advanced or metastatic disease (FIGO stages III and IV) (1-3). Although initial therapy with surgery and chemotherapy may be effective, the majority of patients suffer relapse, often resulting in drug resistance. Thus, identifying diagnostic markers and developing novel therapeutic strategies are important challenges to combat EOC.

In our previous study, we successfully inhibited the proliferation of EOC cells in vitro and in a xenograft model, using a novel triazole nucleoside analog, which led to suppressed expression of heat shock factor 1 (HSF1) (4). HSF1 is the crucial transcription factor of molecular chaperones and heat shock proteins, which is a highly conserved mechanism to protect organisms against various stresses. The cytoprotective and anti-apoptotic activities of HSF1 have been well documented, and research concerning its physiological function has mainly focused on its regulatory roles in the expression of chaperone genes $(5,6)$. Nevertheless, in the past few years, the capacity of HSF1 to orchestrate a genome-wide transcriptional program has attracted growing interest, and HSF1 has revealed an important role in multiple physiological processes (7-9).

In both human cancer cell lines and tumor tissues of various origins, including breast, colon, lung, cervix, pancreatic, prostate and mesenchymal tumors, highly amplified HSF1 was detected, while low expression of the protein was observed in normal cells (9-15). There is a growing understanding of the carcinogenesis-promoting role of HSF1, and its overexpression has been found to be associated with reduced survival $(7,8,11,16-19)$. More importantly, HSF1 orchestrates a genomic transcriptional program to support malignant transformation. Cancer cells show a large dependency on HSF1 
to maintain proliferation and survival compared with normal cells $(7,9,19)$. Additionally, HSF1 was found to regulate more genes in highly malignant cancer cells than in less aggressive or non-transformed cells, and many of these genes are cancerspecific (7). Therefore, HSF1 is regarded as an attractive target for cancer therapy $(16,20-23)$. Despite the well-uncovered physiological roles of HSF1 in diverse tumor types, its expression pattern and carcinogenesis function in EOC remain elusive.

Recently, we reported a nucleoside analog that attenuated the proliferative activity and tumorigenesis ability of EOC cells by inhibition of HSF1 expression (4). The finding motivated us to define the role of HSF1 in ovarian carcinogenesis and to address the correlation between inhibition of the HSF1 expression and the antitumor effect in EOC. The objective of present study included the investigation of the HSF1 expression pattern in EOC tissues and the antitumor effect of HSF1 in EOC cells.

\section{Materials and methods}

Tissue collection and immunohistochemical assay. Healthy and EOC ovarian tissue blots were purchased from Alenabio (Xi'an, China) and Shanghai Outdo Biotech Co., Ltd. (Shanghai, China). The collected tissues included 42 benign (30 serous cystadenoma, 12 mucinous cystadenoma), 9 borderline ( 7 serous adenocarcinoma, 2 mucinous adenocarcinoma), 126 malignant (88 serous adenocarcinoma, 11 mucinous adenocarcinoma, 17 endometrioid adenocarcinoma, 10 clear cell carcinoma) and 62 healthy ovarian tissues. The stages and histological grades were established according to the FIGO criteria.

A rabbit polyclonal antibody (4356; Cell Signaling Technology, Inc., Danvers, MA, USA) using a Histostain-Plus IHC kit (MRBiotech, Shanghai, China) was used to detect HSF1 protein. Following 3,3'-diaminobenzidine (DAB) staining and hematoxylin counterstaining, the immunostained sections were scrutinized using light microscopy. Staining extent was calculated by a semi-quantitative scoring system, and the percentage of stained cells was scored as follows: $1(<25 \%), 2(25-49 \%), 3(50-75 \%)$ and $4(>75 \%)$; and the staining intensity was subjectively estimated as: $1(+), 2(++)$ and $3(+++)$. The final scores are presented as the 'percentage of stained cells' $x$ 'staining intensity'. Depending on the expression score, the specimens were grouped as negative (0), low expression (1-5) and high expression (6-12).

The Mann-Whitney U test and t-test were used for statistical analysis; $\mathrm{p}<0.05$ was considered statistically significant. Statistical analysis was performed using SPSS statistical software (SPSS, Inc., Chicago, IL, USA).

Cell culture and infection. Human EOC cell line SKOV3 was purchased from the Shanghai Cell Bank of the Chinese Academy of Sciences (Shanghai, China). SKOV3 and its derivatives were maintained in McCoy's 5A medium (SigmaAldrich, St. Louis, MO, USA) with $10 \%$ fetal bovine serum (FBS; Gibco, Carlsbad, CA, USA) at $37^{\circ} \mathrm{C}$ with $5 \% \mathrm{CO}_{2}$.

Short hairpin RNAs (shRNAs) against HSF1 and the corresponding expression vectors were designed and constructed by GenePharma (Shanghai, China). Lentiviral particles, expressing specific shRNAs against HSF1, were also prepared by GenePharma. SKOV3 cells were infected with lentiviral particles expressing the scramble shRNA or shRNA against
HSF1 in the presence of $5 \mu \mathrm{g} / \mathrm{ml}$ polybrene, generating SKOV3-NC and SKOV3-shHSF1 cells, respectively. SKOV3-NC and SKOV3-shHSF1 clones were cultured and screened with puromycin $(1 \mu \mathrm{g} / \mathrm{ml}$; Merck Millipore, Billerica, MA, USA) after infection. The HSF1 inhibitory effect was examined at both the mRNA and protein levels.

Cell proliferation assessment. 3-(4,5-Dimethylthiazol-2-yl)2,5-diphenyltetrazolium bromide (MTT; Sigma-Aldrich) assay was employed to assess the cell viability and to determine cell proliferative activity. The assay was performed according to a previously published procedure (4). Briefly, $\sim 1 \times 10^{4}$ cells were seeded per well in a 96-well plate, and incubated in $100 \mu 1$ medium. Fresh medium was added every $48 \mathrm{~h}$. For cell viability assessment, $10 \mu \mathrm{l}$ MTT ( $5 \mathrm{mg} / \mathrm{ml})$ was added, followed by a 4-h incubation at $37^{\circ} \mathrm{C}$. The medium was replaced by $150 \mu \mathrm{l}$ dimethyl sulfoxide (DMSO; Sigma-Aldrich), and absorbance at $490 \mathrm{~nm}$ was determined in a microplate reader (Bio-Rad Laboratories, Inc., Hercules, CA, USA). Each assay contained at least six wells and all experiments were independently repeated at least 3 times.

Flow cytometric analysis. To determine the cell cycle distribution, cells in the logarithmic phase were dissociated by $0.25 \%$ trypsin-ethylenediaminetetraacetic acid (Life Technologies, Carlsbad, CA, USA), seeded in a 6-well plate and then allowed to attach and proliferate for $24 \mathrm{~h}$. Cells were harvested by trypsin digestion, rinsed with phosphate-buffered saline (PBS), and fixed with $70 \%$ cold-ethanol for at least $12 \mathrm{~h}$ at $-20^{\circ} \mathrm{C}$. The resultant cells were centrifuged and resuspended, and were treated with RNase A for $30 \mathrm{~min}$ at $37^{\circ} \mathrm{C}$. Then propidium iodide (PI) was added and incubated at $4^{\circ} \mathrm{C}$ for $30 \mathrm{~min}$.

Annexin V-PE/7-AAD Apoptosis Detection kit (KeyGen, Nanjing, China) was used to evaluate cell apoptosis. Cells in a logarithmic phase were dissociated and seeded in $6-\mathrm{cm}$ dish. After a 48-h culture, the resulting cells were harvested by trypsin digestion, and analyzed by following the manufacturer's instructions.

The flow cytometric detection and data analysis were performed with a flow cytometer (BD Accuri ${ }^{\mathrm{TM}}$ C6; BD Biosciences, Franklin Lakes, NJ, USA).

RNA extraction and quantitative PCR. RNA preparation and quantitative PCR were performed as described in the literature $(4,24)$. Cells were harvested and rinsed, and RNA was extracted with TRIzol reagent (Life Technologies) according to the manufacturer's instructions. Genomic DNA contamination was removed by treatment with DNase I (Fermentas, Hanover, MD, USA). Reverse-transcription was performed with M-MLV Reverse Transcriptase (Promega, Madison, WI, USA). Quantitative PCR was performed with the SYBR Real-Time PCR kit (GenePharma) on a Mastercycler ep realplex (Eppendorf, Hamburg, Germany).

DNA oligo pairs used in this study were as follows: GAPDH forward, GAGTCAACGGATTTGGTCGT and reverse, TTG ATTTTGGAGGGATCTCG; HSF1 forward, CATGAAGCAT GAGAATGAGGCT and reverse, ACTGCACCAGTGAGATC AGGA. Relative RNA concentration was calculated from cycle thresholds and GAPDH was used as the internal standard control. Three independent experiments were performed. 
A Normal ovarian tissue

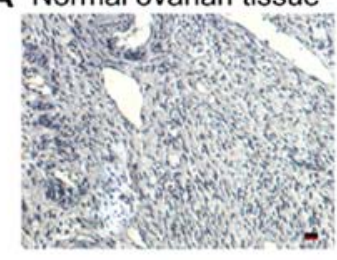

B Benign serous cystadenoma

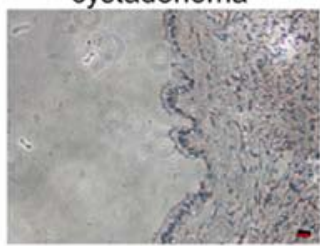

Benign mucinous cystadenoma

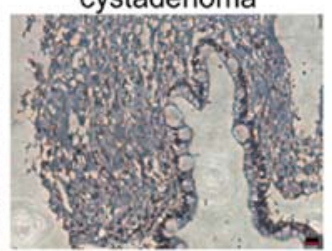

C Borderline serous adenocarcinoma

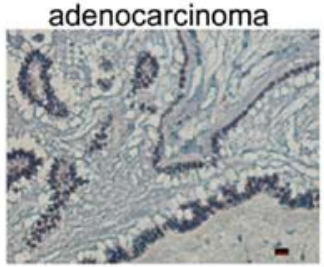

Borderline mucinous adenocarcinoma

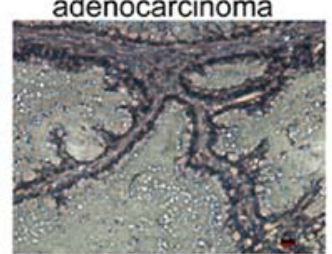

D Malignant serous adenocarcinoma
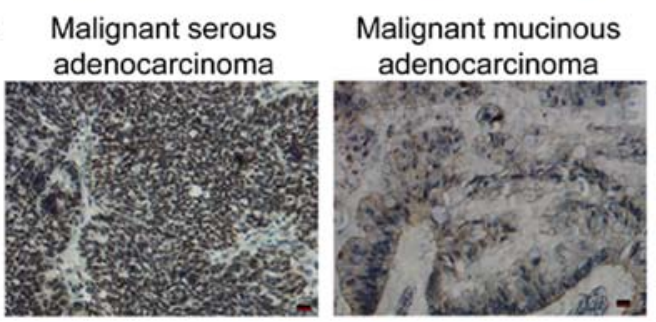

Malignant endometrioid adenocarcinoma

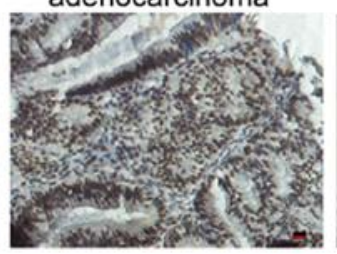

Malignant clear cell carcinoma

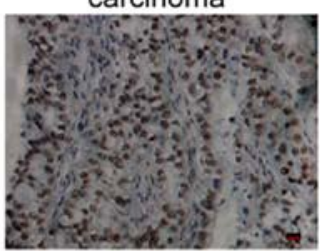

Figure 1. Expression of heat shock factor 1 (HSF1) in epithelial ovarian cancer (EOC) tissues. HSF1 protein was detected by immunohistochemical analysis in (A) healthy (normal) ovarian tissues; (B) benign serous cystadenoma, mucinous cystadenoma; (C) borderline serous adenocarcinoma, borderline mucinous adenocarcinoma; and (D) malignant serous adenocarcinoma, mucinous adenocarcinoma, endometrioid adenocarcinoma and clear cell carcinoma tissues. HSF1 was detected with specific antibodies against HSF1 and visualized with 3,3'-diaminobenzidine (DAB) staining and hematoxylin counterstaining.

Immunoblot assay. Routine western blot analysis was carried out according to a previously a published protocol (4). Anti-human HSF1 rabbit polyclonal antibody (4356; Cell Signaling Technology,Inc.) and anti-human $\beta$-actin mouse monoclonal antibody (A5441; Sigma-Aldrich) were used as primary antibodies. Horseradish peroxidase (HRP)-conjugated antibodies (goat anti-rabbit IgG-HRP, 111-035-003; goat anti-mouse IgG-HRP, 115-035-003; Jackson ImmunoResearch Inc., West Grove, PA, USA) were applied as secondary antibodies. Immunoblot signals were visualized with SuperSignal West Pico chemiluminescent substrate (Thermo Fisher Scientific, Inc., Rockford, IL, USA).

Xenograft tumor construction. Animal maintenance and experiments were performed in compliance with the guidelines of the Institutional Animal Care and Use Committee of Shanghai Jiaotong University (Shanghai, China). SKOV3-NC and SKOV3-shHSF1 cells in a logarithmic phase were harvested by trypsin digestion and washed twice with PBS. Cells $\left(1 \times 10^{6}\right)$ were injected subcutaneously into 4-week-old female BALB/c nude mice. After 2 weeks, the tumor growth of SKOV3-NC cells was well-established. The mouse weight and tumor size were monitored every 3 or 4 days. At the end of the experiment, the mice were sacrificed by cervical dislocation, and tumors were excised.

\section{Results}

HSF1 expression is elevated in malignant EOC tissues. Hyperexpression of HSF1 is considered to be an aggressiveness marker in certain malignant carcinomas. However, to the best of our knowledge the clinical and prognostic significance of HSF1 expression in ovarian neoplasms has not been well studied. In order to investigate whether HSF1 is elevated in EOC patients, the HSF1 expression pattern was determined in normal ovarian tissues and EOC tissue samples, which were derived from patients.

We investigated the relationship of HSF1 in EOC to clinical parameters in a series of primary tumors. Forty-two benign (30 serous cystadenoma, 12 mucinous cystadenoma), 9 borderline (7 serous adenocarcinoma, 2 mucinous adenocarcinoma), 126 malignant (88 serous adenocarcinoma, 11 mucinous adenocarcinoma, 17 endometrioid adenocarcinoma, 10 clear cell carcinoma) and 62 healthy ovarian tissues were included.

The expression of HSF1 was examined by immunochemical staining. As shown in Fig. 1A, HSF1 was barely detected in the normal ovarian tissues. The expression of HSF1 was low and largely restricted in the infiltrating stroma and tumor areas bordering necrosis in the benign and border- 
Table I. The HSF1 expression patterns in EOC tissues.

\begin{tabular}{|c|c|c|c|}
\hline Clinicopathological characteristics & $\begin{array}{l}\text { No HSF1 } \\
\text { n (\%) }\end{array}$ & $\begin{array}{c}\text { Low HSF1 } \\
\text { n (\%) }\end{array}$ & $\begin{array}{c}\text { High HSF1 } \\
\text { n (\%) }\end{array}$ \\
\hline Normal ovarian tissue & $60(96.77)$ & $2(3.23)$ & $0(0.00)$ \\
\hline \multicolumn{4}{|l|}{ Serous } \\
\hline Benign serous cystadenoma $^{a}$ & $9(30.00)$ & $21(70.00)$ & $0(0.00)$ \\
\hline Borderline serous adenocarcinoma $^{a}$ & $3(42.86)$ & $4(57.14)$ & $0(0.00)$ \\
\hline Malignant serous adenocarcinoma ${ }^{\mathrm{a}}$ & $29(32.95)$ & $42(47.73)$ & $17(19.32)$ \\
\hline \multicolumn{4}{|l|}{ Mucinous } \\
\hline Benign mucinous cystadenoma & $11(91.67)$ & $1(8.33)$ & $0(0.00)$ \\
\hline Borderline mucinous adenocarcinoma & $1(50.00)$ & $1(50.00)$ & $0(0.00)$ \\
\hline Malignant mucinous adenocarcinoma ${ }^{\mathrm{a}, \mathrm{c}}$ & $4(36.36)$ & $6(54.55)$ & $1(9.09)$ \\
\hline \multicolumn{4}{|l|}{ Endometrioid } \\
\hline Malignant endometrioid adenocarcinoma ${ }^{\mathrm{b}}$ & $12(70.59)$ & $3(17.65)$ & $2(11.76)$ \\
\hline \multicolumn{4}{|l|}{ Clear cell } \\
\hline Malignant clear cell carcinoma ${ }^{\mathrm{a}}$ & $1(10.00)$ & $4(40.00)$ & $5(50.00)$ \\
\hline
\end{tabular}

line tumors (Fig. 1B and C), while dense and massive HSF1 staining was observed in the malignant EOC tissues (Fig. 1D).

A semi-quantification scoring system was used to quantify the HSF1 expression pattern. The expression patterns of HSF1 in EOC tissues with different clinicopathological features are listed in Table I. Correlation of the HSF1 status with clinical parameters was further delineated by Mann-Whitney U test analysis. Compared with the normal ovarian tissues, malignant EOC tissues, including serous, mucinous, endometrioid and clear cell, showed significantly elevated HSF1 expression. High HSF1 expression was detected in malignant tissues only, including serous, mucinous, endometrioid and clear cell carcinoma. This suggests an association between high HSF1 expression and a more malignant phenotype.

In Fig. 2A-D the HSF1 staining scores are shown in serous, mucinous, endometrioid and clear cell EOC tissues, respectively. The HSF1 expression was significantly higher in benign serous tissues and serous EOC tissues, including borderline and malignant, than the normal ovarian tissues (Fig. 2A). Compared with the benign serous cystadenoma, malignant serous adenocarcinoma showed higher HSF1 staining scores. However, there was no significant difference between the benign and borderline tissues, or the borderline and malignant tissues. In malignant mucinous adenocarcinoma, the HSF1 expression was significantly elevated (Fig. 2B), and the HSF1 staining score was higher in malignant carcinoma than that in the corresponding benign tumors. Endometrioid (Fig. 2C) and clear cell (Fig. 2D) carcinoma also had higher expression of HSF1 than that in the normal ovarian tissues.

The results showed that HSF1 is activated in the malignant state, suggesting HSF1 to be a candidate biomarker for EOC, and implying its biological significance in ovarian malignancy. The elevated expression of HSF1 also suggests it as a therapeutic target against EOC, and motivated us to examine whether targeting HSF1 leads to an anticancer effect in EOC cells.
HSF1 knockdown leads to reduced growth and induced apoptosis in SKOV3 cells. As discussed above, HSF1 represents an attractive therapeutic target for several human cancers, and our data confirmed its elevated expression in EOC. Thus, we aimed to ascertain whether targeting HSF1 leads to antitumor activities in EOC. To demonstrate the potential effect of targeting HSF1 in EOC treatment, we firstly investigated the biological function of HSF1 knockdown in SKOV3 cells, which is an EOC-derived immortalized cell line.

In the attempt to obtain stable HSF1-knockdown SKOV3 cells, scramble and HSF1-specific shRNA lentiviral particles were generated and transfected into SKOV3 cells, generating SKOV3-NC and SKOV3-shHSF1 cell lines respectively. Both mRNA and protein expression levels of HSF1 were examined to confirm the silencing efficiency. As expected, the expression of HSF1 was silenced in the SKOV3-shHSF1 cells, while its expression remained the same in the SKOV3-NC cells (Fig. 3A and B).

To assess the significance of targeting HSF1 in cell proliferation regulation, cell viability was determined by MTT assay, and a growth curve was evaluated for the SKOV3-NC and SKOV3-shHSF1 cells. Notably, a reduction in proliferation was observed in the SKOV3-shHSF1 cells (Fig. 3C), indicating that downregulation of HSF1 led to inhibition of cell growth.

Cell cycle distribution and cell apoptosis were investigated in the SKOV3-shHSF1 and SKOV3-NC cells. The percentage of early apoptotic cells was markedly increased in the SKOV3-shHSF1 cells (11.87\%), relative to that in the SKOV3-NC cells $(6.47 \%)$ (Fig. 4A and B). Additionally, the percentage of late apoptotic cells was slightly increased in the SKOV3-shHSF1 group (Fig. 4A). This implies that downregulation of HSF1 in the SKOV3 cells led to induction of cell apoptosis.

Then, cell cycle distribution was investigated in both SKOV3-shHSF1 and SKOV3-NC cells. As illustrated in 

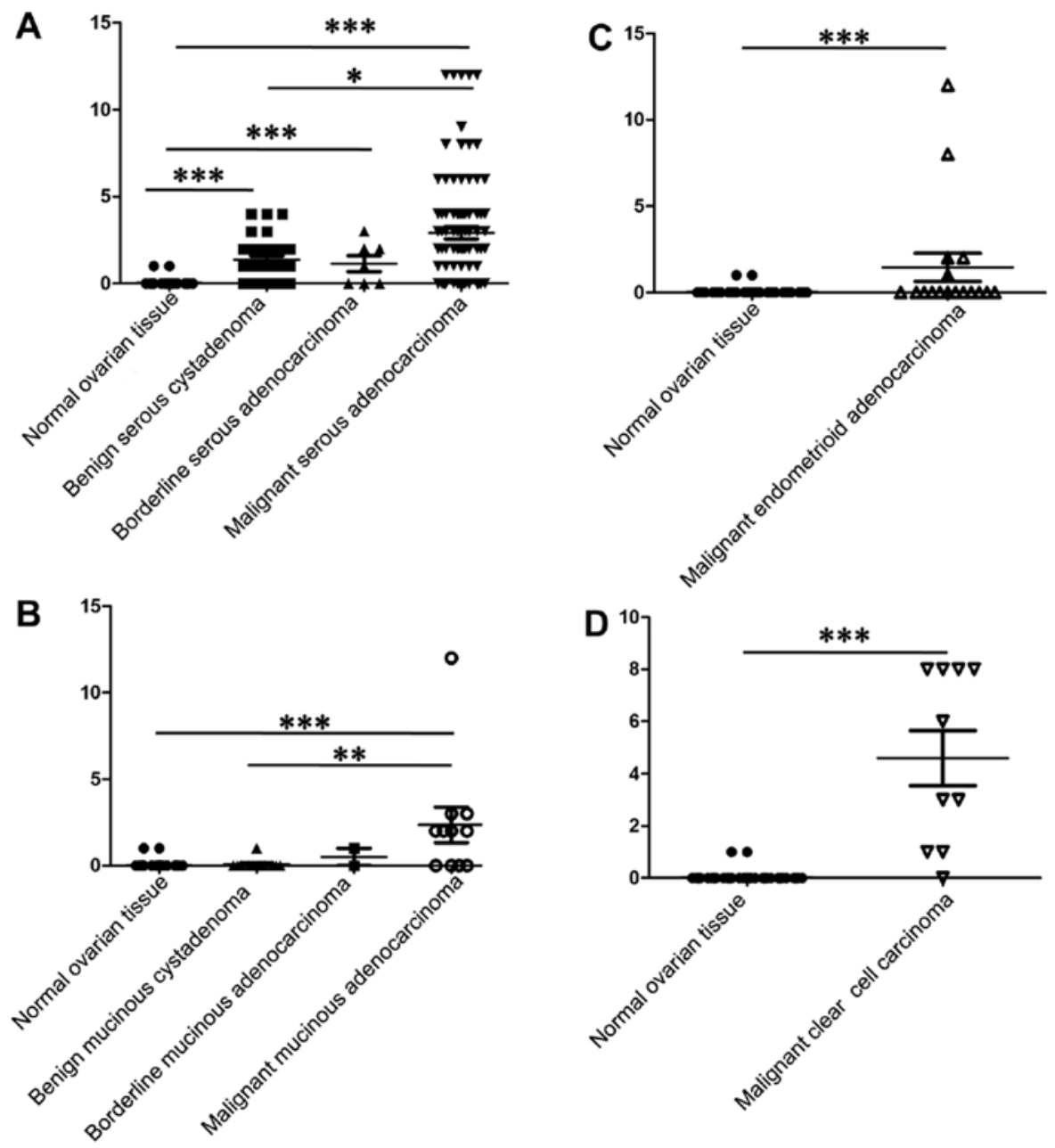

Figure 2. Relationship between heat shock factor 1 (HSF1) expression and clinical features of the epithelial ovarian cancer (EOC) cases. HSF1 expression was evaluated with a semi-quantification scoring system considering both the positive percentage and staining intensity. Bars, median values. Statistical analyses were performed by Mann-Whitney U test, and statistical significance is indicated $\left({ }^{*} \mathrm{p}<0.05,{ }^{* *} \mathrm{p}<0.01,{ }^{* * *} \mathrm{p}<0.001\right)$.
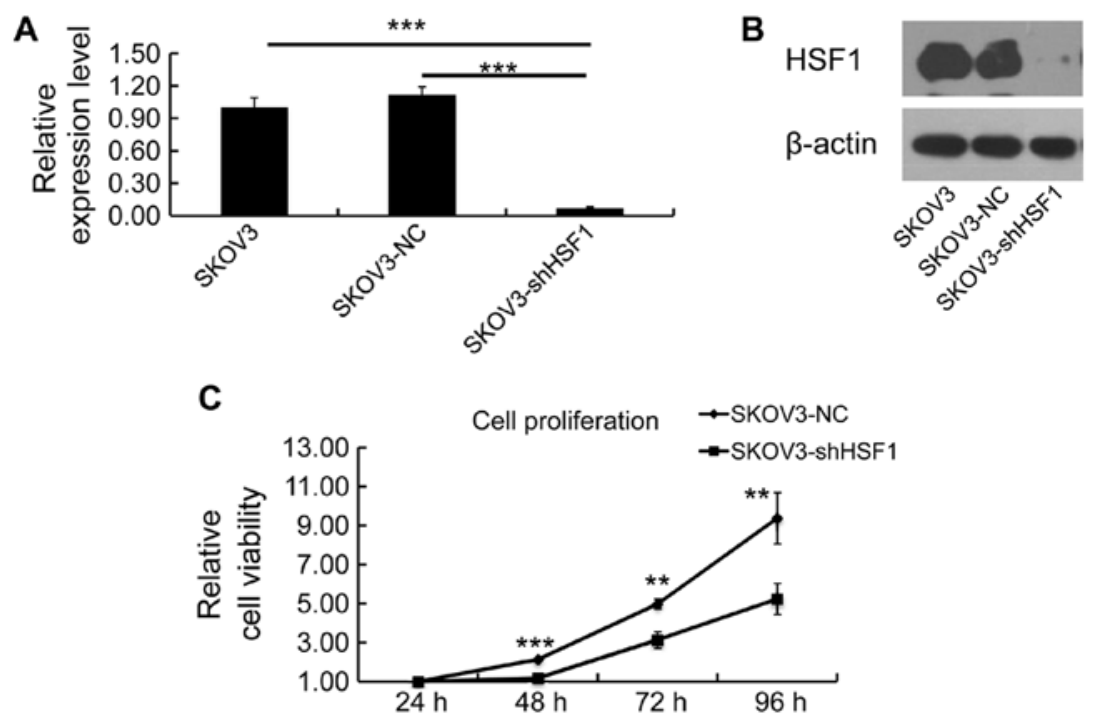

Figure 3. Characterization of SKOV3 cells with stable suppression of heat shock factor 1 (HSF1). (A) Quantitative PCR was employed to examine the transcription level of HSF1. GAPDH was used as the internal standard. The relative transcript concentration was normalized to original SKOV3 cells. Results are the average of three independent experiments, and bars indicate the standard deviations (SDs) $\left.{ }^{* * *} \mathrm{p}<0.001\right)$. p-values were calculated by t-test. (B) Western blot analysis was used to evaluate the protein expression of HSF1 in HSF1 stable knockdown SKOV3 cells (SKOV-shHSF1), scramble shRNA stably transfected SKOV3 cells (SKOV3-NC) and parental SKOV3 cells (SKOV3). $\beta$-actin was employed as the loading reference. (C) Cell proliferation of SKOV3-NC and SKOV3-shHSF1 cells was examined by MTT assay. Cells $\left(1 \times 10^{4}\right)$ in a logarithmic phase were seeded in fresh culture medium and allowed to grow for 24,48 , 72 or 96 h. Each experiment was repeated in triplicate, and error bars indicate the SDs $\left(* \mathrm{p} p<0.01,{ }^{* * *} \mathrm{p}<0.001\right)$. 

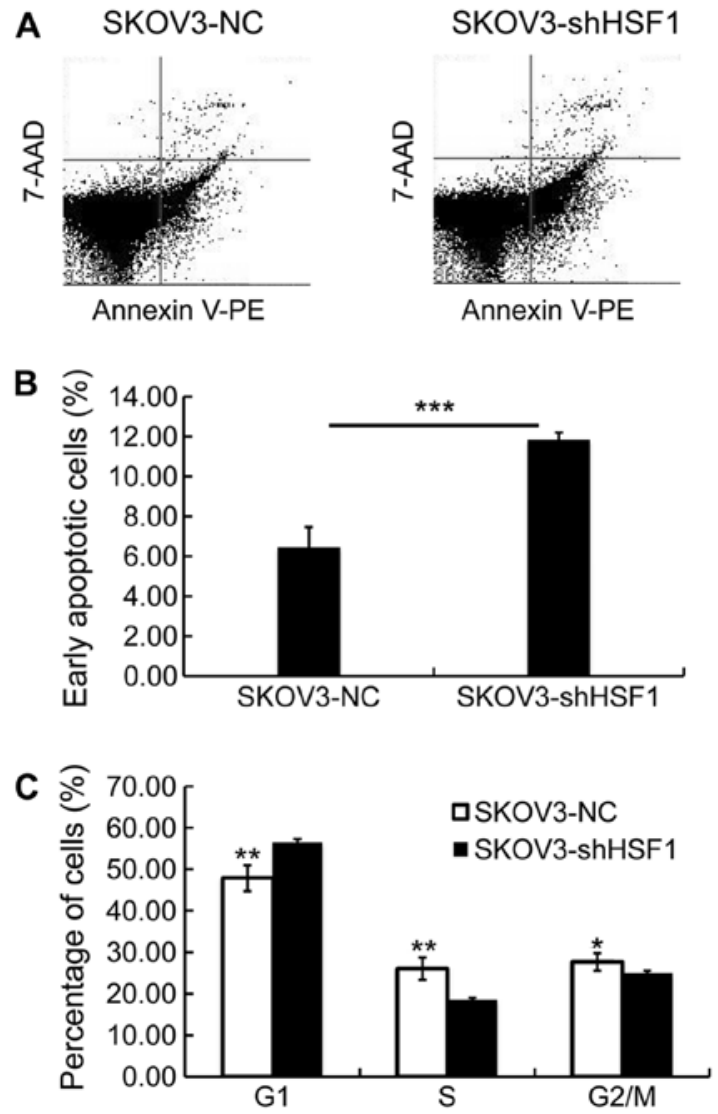

Figure 4. Effects of the downregulation of heat shock factor 1 (HSF1) on cell apoptosis and cell cycle distribution. (A) Cells in a logarithmic phase were seeded and allowed to grow for $48 \mathrm{~h}$ before harvesting. Then cell samples were prepared for flow cytometric analysis. Annexin V-PE was used to label the apoptotic cells and 7-AAD was used to stain the necrotic cells. Annexin V-PE(+)/7-AAD(-) indicates early apoptotic cells, while Annexin V-PE(+)/7-AAD(+) indicates late apoptotic cells. The images represent one of three independent experiments, which show similar results. (B) The early apoptotic cell percentage was calculated in SKOV3-NC and SKOV3shHSF1 cells. Error bars indicate the standard deviations (SDs) $\left({ }^{* * *} \mathrm{p}<0.001\right)$. (C) Cell cycle distribution analysis was performed by flow cytometry. Cells were harvested at $24 \mathrm{~h}$ after cell passage, and propidium iodide (PI) was used to stain cellular DNA. Numbers indicate the means \pm SD cell percentage of three experiments, and error bars indicate the SDs $(* * \mathrm{p}<0.01)$.

Fig. 4C, the percentage of cells at the G1 phase was increased in the SKOV3-shHSF1 cells, while the percentages of $\mathrm{S}$ and G2/M phase cells were decreased in the SKOV3-shHSF1 cells. The observed differences in cell cycle distribution confirmed that the suppression of proliferative activity resulted from HSF1 knockdown. These results demonstrated the antitumor effect of targeting HSF1 against EOC in vitro.

Depletion of HSF1 in SKOV3 cells results in reduced tendency of carcinogenesis. An animal study was performed to confirm the antitumor activity arising from the downregulation of HSF1 in vivo. After injection into immunodeficiency nude female mice for 14 days, the control cells formed noticeable tumors. However, the HSF1-depleted cells (SKOV3-shHSF1 cells) exhibited no tumor genesis until 39 days post-injection (Fig. 5A). In addition, the body weights of the mice were also recorded, and no discernible weight alteration was observed between the two groups (Fig. 5B). These data revealed that targeting HSF1 in SKOV3 cells also led to
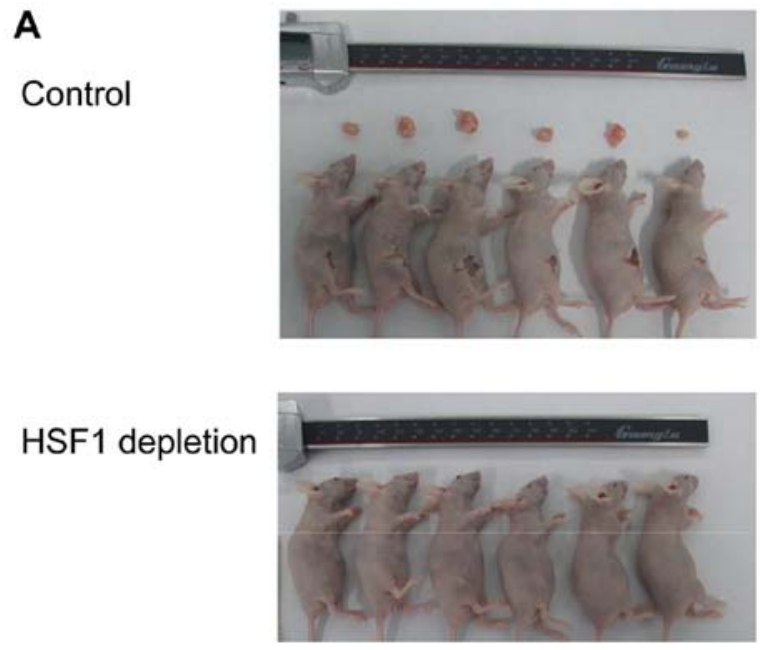

B

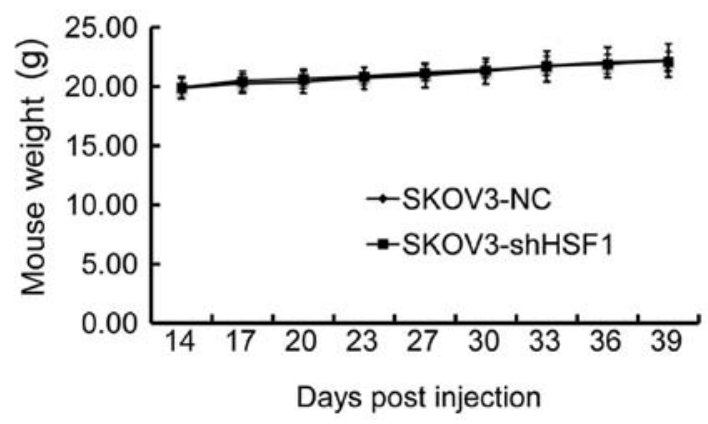

Figure 5. The effect of heat shock factor 1 (HSF1) knockdown on carcinogenesis activity. (A) Images of xenografted mice and tumor tissues. (B) During carcinogenesis, the body weight of mice implanted with SKOV3-NC or SKOV3-shHSF1 cells was monitored. Results represent the mean weights of mice $(n=6)$. Error bars indicate standard deviations (SDs).

an antitumor effect in vivo, suggesting that HSF1 may be used as a potential therapeutic target against EOC.

\section{Discussion}

The biological functions of HSF1 in promoting cell protection and survival against stress have been well understood for decades. In addition, it is believed that the regulatory function of HSF1 is performed by coordinating chaperone protein expression. However, in the past few years, the biological function of HSF1 has been found to be more extensive than previously assumed, especially in regards to tumor cells $(7,9,11)$. Increasing evidence indicates this ancient transcriptional regulator to be a genome-wide regulator of carcinogenesis and tumor progression. The critical cellular roles of HSF1 include maintaining or promoting tumorigenesis, facilitating tumorigenesis and malignant transformation, maintaining protein homostasis and hindering cell apoptosis. More importantly, several lines of evidence suggest that downregulation of HSF1 is a promising antitumor strategy for several types of malignancies. Therefore, HSF1 has been regarded as a promising candidate target for cancer therapy (20-23), and strategies that are capable of modulating HSF1 expression to elicit anticancer activity have recently raised considerable interest (25). However, data concerning the expression pattern of HSF1 in human ovarian cancer, 
and the antitumor effect of HSF1 modulation against human ovarian cancer have not been investigated.

Our previous study demonstrated that suppression of HSF1 expression, probed by a nucleoside analog, efficiently inhibited the proliferation activity and tumorigenesis in EOC-derived cells (4), suggesting the potential to combat EOC by downregulation of HSF1 expression. In the present study, we evaluated the HSF1 expression pattern in human EOC tissues, and investigated the antitumor function of the attenuated expression of HSF1 in EOC-derived cell lines (SKOV3). We observed that HSF1 was highly expressed in malignant serous, mucinous, endometrioid, and clear cell ovarian carcinoma and low HSF1 expression was detected in benign serous and mucinous cystadenoma. In contrast, HSF1 was barely detected in healthy ovarian tissues.

The observed overexpression of HSF1 in human EOC tissues led us to suggest HSF1 as a molecular target against ovarian cancer. Additionally, the present study showed that HSF1 knockdown induced early apoptosis and inhibited proliferative activity in SKOV3 cells. Moreover, downregulation of HSF1 was found to result in suppression of EOC carcinogenesis.

Taken together, our results demonstrated that HSF1 expression plays an important role in the malignant potential of human EOC and HSF1 may be a promising diagnotic biomarker for malignant epithelial ovarian tumors. Furthermore, our data showed the potential value of manipulating HSF1 as a therapeutic strategy against EOC. In conclusion, our findings, although still preliminary, provided critical information concerning the understanding of the cellular function of HSF1 in ovarian cancer. This study may facilitate the early diagnosis and efficacious treatment of human EOC.

\section{Acknowledgements}

This study was supported by grants from the National Natural Science Foundation of China (nos. 81401216 and 81603189), Shanghai Municipal Health and Family Planning Commission (no. 20134Y128), Shanghai Jiaotong University School of Medicine (no. 13XJ10067), and Shanghai Committee of Science and Technology (no. 14YF1408200), as well as the Innovation Team of Natural Science Foundation of the Department of Education of Guizhou Province (no. QJHRCTDZ[2015]57).

\section{References}

1. Coward JI, Middleton K and Murphy F: New perspectives on targeted therapy in ovarian cancer. Int $\mathrm{J}$ Womens Health 7: 189-203, 2015.

2. Syrios J, Banerjee S and Kaye SB: Advanced epithelial ovarian cancer: from standard chemotherapy to promising molecular pathway targets - where are we now? Anticancer Res 34 2069-2077, 2014.

3. Landriscina M, Amoroso MR, Piscazzi A and Esposito F: Heat shock proteins, cell survival and drug resistance: the mitochondrial chaperone TRAP1, a potential novel target for ovarian cancer therapy. Gynecol Oncol 117: 177-182, 2010.

4. Chen YF, Dong Z, Xia Y, Tang J, Peng L, Wang S and Lai D: Nucleoside analog inhibits microRNA-214 through targeting heat-shock factor 1 in human epithelial ovarian cancer. Cancer Sci 104: 1683-1689, 2013.
5. Anckar J and Sistonen L: Regulation of HSF1 function in the heat stress response: implications in aging and disease. Annu Rev Biochem 80: 1089-1115, 2011.

6. Vihervaara A and Sistonen L: HSF1 at a glance. J Cell Sci 127: 261-266, 2014.

7. Mendillo ML, Santagata S, Koeva M, Bell GW, Hu R, Tamimi RM, Fraenkel E, Ince TA, Whitesell L and Lindquist S: HSF1 drives a transcriptional program distinct from heat shock to support highly malignant human cancers. Cell 150: 549-562, 2012.

8. Home T, Jensen RA and Rao R: Heat shock factor 1 in protein homeostasis and oncogenic signal integration. Cancer Res 75: 907-912, 2015.

9. Dai C, Whitesell L, Rogers AB and Lindquist S: Heat shock factor 1 is a powerful multifaceted modifier of carcinogenesis. Cell 130: 1005-1018, 2007.

10. Calderwood SK: HSF1, a versatile factor in tumorogenesis. Curr Mol Med 12: 1102-1107, 2012.

11. Ciocca DR, Arrigo AP and Calderwood SK: Heat shock proteins and heat shock factor 1 in carcinogenesis and tumor development: an update. Arch Toxicol 87: 19-48, 2013.

12. Meng L, Gabai VL and Sherman MY: Heat-shock transcription factor HSF1 has a critical role in human epidermal growth factor receptor-2-induced cellular transformation and tumorigenesis. Oncogene 29: 5204-5213, 2010.

13. Santagata S, Hu R, Lin NU, Mendillo ML, Collins LC, Hankinson SE, Schnitt SJ, Whitesell L, Tamimi RM, Lindquist S, et al: High levels of nuclear heat-shock factor 1 (HSF1) are associated with poor prognosis in breast cancer. Proc Natl Acad Sci USA 108: 18378-18383, 2011.

14. Jin X, Moskophidis D and Mivechi NF: Heat shock transcription factor 1 is a key determinant of $\mathrm{HCC}$ development by regulating hepatic steatosis and metabolic syndrome. Cell Metab 14: 91-103, 2011.

15. Fang F, Chang R and Yang L: Heat shock factor 1 promotes invasion and metastasis of hepatocellular carcinoma in vitro and in vivo. Cancer 118: 1782-1794, 2012.

16. Li Y, Xu D, Bao C, Zhang Y, Chen D, Zhao F, Ding J, Liang L, Wang Q, Liu L, et al: MicroRNA-135b, a HSF1 target, promotes tumor invasion and metastasis by regulating RECK and EVI5 in hepatocellular carcinoma. Oncotarget 6: 2421-2433, 2015.

17. Santagata S, Mendillo ML, Tang YC, Subramanian A, Perley CC, Roche SP, Wong B, Narayan R, Kwon H, Koeva M, et al: Tight coordination of protein translation and HSF1 activation supports the anabolic malignant state. Science 341: 1238303, 2013.

18. Scherz-Shouval R, Santagata S, Mendillo ML, Sholl LM, BenAharon I, Beck AH, Dias-Santagata D, Koeva M, Stemmer SM, Whitesell L, et al: The reprogramming of tumor stroma by HSF1 is a potent enabler of malignancy. Cell 158: 564-578, 2014.

19. Dai C and Sampson SB: HSF1: guardian of proteostasis in cancer. Trends Cell Biol 26: 17-28, 2016.

20. Whitesell L and Lindquist S: Inhibiting the transcription factor HSF1 as an anticancer strategy. Expert Opin Ther Targets 13: 469-478, 2009.

21. Kumar S, Tomar MS and Acharya A: HSF1-mediated regulation of tumor cell apoptosis: a novel target for cancer therapeutics. Future Oncol 9: 1573-1586, 2013.

22. Chen Y, Chen J, Loo A, Jaeger S, Bagdasarian L, Yu J, Chung F, Korn J, Ruddy D, Guo R, et al: Targeting HSF1 sensitizes cancer cells to HSP90 inhibition. Oncotarget 4: 816-829, 2013.

23. Rossi A, Ciafrè S, Balsamo M, Pierimarchi P and Santoro MG: Targeting the heat shock factor 1 by RNA interference: a potent tool to enhance hyperthermochemotherapy efficacy in cervical cancer. Cancer Res 66: 7678-7685, 2006.

24. Chen YF, Dong Z, Jiang L, Lai D and Guo L: Mouse primed embryonic stem cells could be maintained and reprogrammed on human amnion epithelial cells. Stem Cells Dev 22: 320-329, 2013.

25. Agarwal T, Annamalai N, Khursheed A, Maiti TK, Arsad HB and Siddiqui $\mathrm{MH}$ : Molecular docking and dynamic simulation evaluation of Rohinitib - Cantharidin based novel HSF1 inhibitors for cancer therapy. J Mol Graph Model 61: 141-149, 2015. 